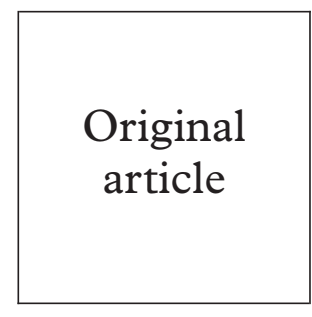

The Population Council, New York, USA

C Coggins

K Blanchard

C Ellertson

Asociacion

Dominicana

Pro-Bienestar de la

Familia, Santo

Domingo, Dominican

Republic

F Alvarez

V Brache

Sydney Centre for Reproductive Health

Research, Department

of Obstetrics and

Gynaecology,

University of Sydney,

Sydney, Australia

E Weisberg

Centers for Disease

Control and

Prevention, Atlanta,

USA; The HIVIAIDS

Collaboration, Chiang

Rai, Thailand

P H Kilmarx

University of Southern

California, Los

Angeles, USA

M Lacarra

D Mishell, Jr

Instituto Chileno de

Medicina

Reproductiva,

Santiago, Chile

R Massai

A Salvatierra

Chiang Rai Hospital, Chiang Rai, Thailand

$\mathrm{P}$ Witwatwongwana

The Population

Council, Bangkok,

Thailand

C Elias

Correspondence to:

Kelly Blanchard, Population

Council, One Dag

Hammarskjold Plaza, New

York, NY 10017, USA

kblanchard@pcjoburg.org.za

Accepted for publication 18 July 2000

\title{
Preliminary safety and acceptability of a carrageenan gel for possible use as a vaginal microbicide
}

\author{
C Coggins, K Blanchard, F Alvarez, V Brache, E Weisberg, P H Kilmarx, M Lacarra,
} R Massai, D Mishell, Jr, A Salvatierra, P Witwatwongwana, C Elias, C Ellertson

Objective: We sought to determine the safety and acceptability of vaginal gel formulation PC-503 among low risk, abstinent women. The active ingredient was $2 \%$ pharmaceutical grade lambda carrageenan, a sulphated polymer that is generally recognised as safe by the US Food and Drug Administration.

Methods: 35 women in five sites applied $5 \mathrm{ml}$ of the PC-503 gel vaginally once a day for 7 days while abstaining from sexual intercourse. Visual vaginal examinations were performed on days 1 , 4 , and 8. STI testing and vaginal pool Gram stain preparations were done on days 1 and 8 . Participants were asked about product acceptability.

Results: 34 of the 35 women enrolled completed 7 days' use. Following product use, five reported mild symptoms including "bladder fullness," "genital warmth," or discomfort, and lower abdominal pain, and one had moderate pale yellow cervical discharge. Using the Nugent criteria, three women had bacterial vaginosis (BV) before and after use; three had BV before but not after, and two had BV after but not before. Most of the women found PC-503 to be pleasant or neutral in feel and smell and considered extra lubrication to be an advantage; however, one third found it to be messy.

Conclusions: Vaginal use of PC-503 gel did not cause significant adverse effects in a small number of low risk, sexually abstinent women. Further testing in larger numbers of sexually active women is planned. A smaller volume of gel may be more acceptable to some women.

(Sex Transm Inf 2000;76:480-483)

Keywords: microbicide; carrageenan

\section{Introduction}

There is an urgent need for alternative HIV/STI prevention methods, including vaginal microbicides $^{12}$ and scientists continue to search for safe and effective vaginal microbicide products. The impetus for this search arises from a recognition that women, who constitute an ever greater proportion of people living with HIV worldwide, are often unable to follow the primary HIV risk reduction strategies promoted by the public health community - mutual monogamy, condom use, and treatment of sexually transmitted infections (STI). Women often rely heavily on their partners for economic wellbeing, and may not be able to abstain from sexual contact. While the women themselves may be monogamous, they cannot control the choices their partners make. Similarly, women are not always able to negotiate condom use successfully. Finally, STI are more likely to be asymptomatic among women and treatment is simply not available in many settings.

One of the most promising classes of potentially microbicidal compounds are sulphated polymers, including carrageenans. Sulphated polymers have been known to block in vitro infection with enveloped viruses (including HIV, HTLV-1, and HSV-2) for many years. ${ }^{3}$ Carrageenans have been extensively evaluated in preclinical studies. ${ }^{4-6}$ Carrageenans are derived from seaweed, and are used extensively in the food, pharmaceutical, and cosmetics industries as lubricants, emulsifiers, and stabilising and dispersing agents. Consequently, carrageenans have a long history of human use, and have been categorised by the US Food and Drug Administration (USFDA) as "generally recognised as safe" (GRAS). ${ }^{7}$ Finally, these compounds are easily acquired in pharmaceutical grade and are inexpensive. A previous vaginal formulation of iota carrageenan, PC213, has undergone phase I testing among women and no adverse effects were found. ${ }^{8}$

In October 1997 the Population Council received approval from the USFDA to conduct a phase I study of PC-503, a gel formulation of $2 \%$ pharmaceutical grade lambda carrageenan in a Carbopol vehicle. The aim of the trial reported here was to determine the potential irritation and acceptability of PC-503 for vaginal use among women.

\section{Study sites}

Seven women were enrolled at each of five clinical sites. Four sites are members of the International Committee for Contraception Research (ICCR) network: the Asociacion Dominicana Pro-Bienestar de la Familia in Santo Domingo, Dominican Republic (site 1); the Sydney Center for Reproductive Health Research in Sydney, Australia (site 2); the Chilean Institute of Reproductive Medicine in Santiago, Chile (site 3); and the University of Southern California in Los Angeles, USA (site 4). The fifth site was in northern Thailand, 
where the protocol was implemented by staff from Chiang Rai Hospital and the HIV/AIDS Collaboration, a joint activity of the Thai Ministry of Public Health and the US Centers for Disease Control and Prevention (CDC) (site 5). This protocol was approved by the Population Council (Protocol No 203) and CDC (Protocol No 1863) institutional review boards, and the ethical review committee of the Thai Ministry of Public Health.

\section{Methods}

The protocol called for women volunteers to apply the gel vaginally once a day for 7 days. Participants were asked to abstain from sexual intercourse for the duration of the study. In this study, participants administered PC-503 vaginally using a prefilled single use syringe applicator containing $5 \mathrm{ml}$ of PC-503 $(100 \mathrm{mg}$ of lambda carrageenan). The first application of PC-503 occurred in the clinic under supervision. Volunteers self administered the remaining six applications at home. All women underwent at least three visual vaginal examinations with a speculum: the first on day 1 at study enrolment before any product use; a second on day 4 and a third on day 8 . Women were asked to return for a clinical examination on day 5 if a lesion or other irritation was noted on day 4, and on day 15 if a lesion or other irritation was noted on day 8. Routine vaginal colposcopy was not included in this protocol. If a lesion was noted, however, colposcopy was performed and lesions were photographed at the discretion of the investigator.

The following microbiological samples were taken at recruitment and day 8 regardless of whether there was clinical suspicion of a sexually transmitted disease: vaginal pool specimen (for Candida albicans, Trichomonas vaginalis, clue cells by wet mount and Gram stain); cervical sample (Neisseria gonorrhoeae, Chlamydia trachomatis) urine sample (culture, pregnancy).

Cervical samples were analysed by either culture or nucleic acid hybridisation test for presence of $N$ gonorrhoeae, and by culture, direct antigen or nucleic acid hybridisation test for presence of $C$ trachomatis, depending upon standard practice at the site.

Gram stains taken at baseline and on day 8 (following 7 days of product use) were sent to a central laboratory in the United States and analysed for presence of bacterial vaginosis (BV) using the Nugent criteria. ${ }^{9}$ A score of 0 to 10 was assigned on the basis of the relative proportion of bacterial morphotypes (large Gram positive rods, small Gram negative or variable rods, or curved rods), with a score of 0 corresponding to the most Lactobacillus predominant vaginal flora and a score of 10 corresponding to a vaginal flora characterised by replacement of lactobacilli by Gardnerella, Bacteroides, and Mobiluncus morphotypes. The criterion for defining presence of $\mathrm{BV}$ is a score of 7 or higher; a score of $4-6$ is considered "intermediate," and a score of 0 to 3 is considered "normal."

\section{Results}

Participant recruitment began in January 1998 and data collection finished in May 1998. A total of 35 healthy women volunteers were enrolled, and all but one completed the study. The woman who did not complete the study (site 2, 006) began her menses early, and inserted six of the seven applications of PC-503. Her final examination was conducted on day 7 instead of day 8; all findings were negative. During follow up one woman reported genital discomfort or itching (site 4 001) and two complained of a burning sensation (site 4006 , site 5 006).

Two women reported transient lower abdominal pain (site 1 006, site 1 009); one woman reported urinary hesitancy (site 1004 ) and two feelings of "full bladder" (site 1009 , site 4 002). One woman reported genital warmth after gel application (site 5 001) and one woman a feeling of "heavy uterus" on the first day of gel use (site 5 005). All symptoms were considered "mild" by the women.

Of the 35 women enrolled in the trial, 32 had completely normal clinical examinations at the baseline, day 4, and 1 week follow up visits. For all women in the study, findings from wet mount examinations ( $C$ albicans, $T$ vaginalis, and clue cells) were negative at baseline, day 4 , and day 8. In addition, all tests for $N$ gonorrhoeae and $C$ trachomatis yielded negative results. No lesions with epithelial disruption were noted. Baseline clinical examination revealed a $1-2 \mathrm{~mm}$ red macule on the ectocervix of one patient (site 5 005) that remained unchanged at the day 4 and day 8 visits. In another patient (site 5 008), Nabothian cysts and a Bartholin's duct cyst were identified at baseline and remained unchanged at the day 4 and 8 visits. These findings were all considered "minor" by the clinical investigators. In only one case did an investigator observe any change in the vagina or cervix that was deemed serious enough to warrant colposcopic examination (site 5 001) and results from this examination revealed a stable finding thought to be an endometriotic rest.

Based on the Nugent criteria, eight of the 35 trial participants had a score at some point during the study that placed them in the "BV" category $(\geqslant 7$ ) (samples were taken at screening and day 8). Three women had evidence of BV at screening but not on day 8 (site 2001 , site 2 005, site 5 006), two did not have evidence of BV at screening but did on day 8 (site 2006 , site 4 003) and three had evidence of BV both at screening and at day 8 (site 1009 , site 3003 , site 5 012).

PRODUCT ACCEPTABILITY

Most of the women in the study considered PC-503 easy to apply, pleasant or neutral in feel and smell, and non-irritating (table 1). Although 23\% reported that the gel caused discomfort or irritation, the question did not differentiate between the two, and in many cases the women were referring only to discomfort due to wetness and did not experience irritation. Nearly two thirds considered a product providing extra lubrication during 
Table $1 \quad P C-503$ acceptability $(n=35)$

\begin{tabular}{lllll}
\hline Product characteristic & Yes & No & Neutral & Don't know \\
\hline Messy to use & $37 \%$ & $54 \%$ & $9 \%$ & \\
Caused discomfort or irritation & $23 \%$ & $74 \%$ & $3 \%$ & \\
Easy to apply & $89 \%$ & $3 \%$ & $9 \%$ & \\
Easy to dispose of & $71 \%$ & $3 \%$ & $6 \%$ & \\
Pleasant feel & $20 \%$ & $29 \%$ & $51 \%$ & \\
Pleasant smell & $17 \%$ & $6 \%$ & $77 \%$ & \multirow{2}{*}{$26 \%$} \\
Could be used without one's partner's knowledge† & $46 \%$ & $29 \%$ & & $23 \%$ \\
Considers extra lubrication to be an advantaget & $66 \%$ & $11 \%$ & & $2 \%$ \\
\hline
\end{tabular}

${ }^{\star_{n}}=28$; participants in the Australia site were not asked about disposal of the product packaging. †Questions on covert use and lubrication provided by the product included "don't know" as a possible response, not "neutral" as included for the other questions.

intercourse to be an advantage. The main negative comment from about one third of the women in the study was that they found PC-503 messy. Several women (including all seven women at the Thai site) reported excessive wetness and runniness following application of the gel, and recommended a reduction in volume.

\section{Discussion}

The data from this phase I preliminary safety study indicate that vaginal use of PC-503 does not cause significant irritation to the female reproductive tract. This observation is based on careful visual examination of the vaginal epithelia and cervical mucosa. The pattern of observed abnormalities does not suggest any consistent association with the use of this lambda carrageenan gel. Similar results were previously found with a vaginal formulation of iota carrageenan. ${ }^{8}$

Because of recent findings describing the possibility that the presence of bacterial vaginosis may increase susceptibility to HIV-1 infection, ${ }^{10-12}$ we paid special attention to evaluating the impact of PC-503 on vaginal flora. Three women in our study had a consistent finding of bacterial vaginosis at screening and day 8 , three had evidence of bacterial vaginosis at screening but not on day 8 , and two women who did not have evidence of bacterial vaginosis at screening did at day 8 . It is difficult to interpret these findings definitively given the small sample. Bacterial vaginosis is a fairly common condition caused by an imbalance in the normal vaginal flora. Consequently it is not surprising that a proportion of women had this condition at baseline. We interpret our study to show that there does not appear to be any significant relation between use of PC-503 and bacterial vaginosis. Given the small sample size of this preliminary study, however, larger expanded safety studies of PC-503 will be required to fully document the potential effects of carrageenan on the vaginal mucosa and flora. Placebo controlled expanded safety studies of a carrageenan microbicide are currently under way in South Africa and Thailand. These larger studies will provide a more precise characterisation of the effects of carrageenan on the vaginal mucosa and flora, and will help put clinical findings and reported complaints in context.

The acceptability data suggest that PC-503 was generally well liked with regard to most criteria except volume. It is noteworthy that while most women in this study considered extra lubrication an advantage (a finding that could be quite different in areas where dry sex is preferred), a significant proportion felt that the product was messy and suggested reducing the volume. All of the women from the Thai site complained the gel was messy, which could reflect different population preferences, although the sample size in this study is too small to draw firm conclusions. It is also important to note that acceptability data from intensive use safety trials may not reflect experiences or opinions in "real life" situations; the study environment is necessarily artificial.

In another study of women's preferences concerning formulations of over the counter spermicides $^{13}$ it was found that some women opt to reduce the volume of product they apply when they feel products are too messy. A similar result was seen in a recent study of vaginal product use among drug involved women in the United States and Puerto Rico. ${ }^{14}$ Deliberate use of less than the recommended volume of product could result in diminished efficacy for a proved product. Consequently, based on these results we are planning to reduce the volume of the formulation before proceeding with further expanded safety trials. The concentration of lambda carrageenan will be increased so that the dose of this active ingredient will remain similar.

On a methodological note, it is interesting that, while the protocol called for colposcopy at the discretion of the investigators in the event of uncertainty concerning the nature of observed lesions, only once was colposcopy used. Even in that instance, colposcopy was used to document a finding at the baseline examination (that is, before product use) and to observe the (lack of) change at the first follow up visit. A previous phase I protocol of a vaginal gel formulation of iota carrageenan (in which four of the five participating sites were the same as in this study) required colposcopy of all participants at baseline and follow up. ${ }^{8}$ In that study, the routine use of colposcopy added little to the documentation of vaginal abnormalities that were potentially product related. There is an ongoing debate about the appropriate role of vaginal colposcopic evaluation in early safety trials of vaginal microbicides. Our experience with two preliminary safety studies of carrageenan formulations suggests that, while colposcopy will always remain a valuable tool for clinicians who may need to investigate further and document an abnormality detected on careful visual examination, the performance of routine colposcopy in early phase trials may be hard to justify given the expense, discomfort to the participants, and the difficulty of interpreting the significance of findings among the small sample sizes typical of preliminary safety trials. Obviously, a larger study that seeks to correlate the presence of colposcopic findings with important clinical end points will ultimately be needed to resolve this important debate.

The authors would like to thank Sharon Hillier for her role in analysing Gram stain specimens. In addition we would like to thank Beverly Winikoff, Elizabeth McGrory, and Barbara Friedland for comments on the draft manuscript. In Thailand we land for comments on the draft manuscript. In Thailand we
would like to thank Dr Jullapong Achalapong, Dr Somboonsak 
Yanpaisarn, Supaporn Korattana, Jitkasem Thongpun and Saithip Nualnouch for their roles in conducting the study. In Fraser and Sue Stuart for their roles in conducting the study.

Contributors: CC, CE, and CE were responsible for the design of the study and writing the protocol; CC and KB took the lead in analysing the data and writing the first draft manuscript; FA VB, EW, PHK, ML, RM, DM Jr, AS, and PW were responsible for managing the study at their sites and participated in the enrolment and evaluation of participants. All of the authors provided comments on the draft manuscript and participated in the interpretation of the data.

1 Stein ZA. HIV prevention: the need for methods women can use. Am F Public Health 1990;80:460-2.

2 Elias CJ, Coggins C. Female-controlled methods to prevent sexual transmission of HIV. AIDS 1996;10(suppl 3):S43-51.

3 Nahmias AAJ, Kibrick S, Bernfeld P. Effect of synthetic and biological polyanions on herpes simplex virus. Proc Soc Exp Biol Med 1964;115:993-996.

4 Zaretzky FR, Pearce-Pratt R, Phillips DM. Sulfated polyanions block Chlamydia trachomatis infection of cervix derived human epithelia. Infect Immunol 1995;63: cervix deri

5 Pearce-Pratt R, Phillips DM. Sulphated polysaccharides inhibit lymphocyte-to-epithelial transmission of HIV. Biol inhibit lymphocyte-to-epit

6 Zacharapoulos V, Phillips DM. Vaginal formulations of carrageenan protect mice from herpes simplex virus infection. Clin Diagn Lab Immunol 1997;4:465-8.
7 United States Food and Drug Administration. GRAS (generally recognized as safe) food ingredients-carrageenan. Report by Informatics, Inc for the US Food and Drug Administration. Springfield VA: National Technical Information Service, 1972.

8 Elias CJ, Coggins C, Alvarez F, et al. Colposcopic evaluation of a vaginal gel formulation of iota-carrageenan. Contraception 1997;57:387-9.

9 Nugent RP, Krohn MA, Hillier SL. Reliability of diagnosing bacterial vaginosis is improved by a standardized method of gram stain interpretation. f Clin Microbiol 1991;29:297301 .

10 Sewankambo N, Gray RH, Wawer MJ, et al. HIV-1 infection associated with abnormal vaginal flora morphology and bacterial vaginosis. Lancet 1997;350:546-50.

11 Taha TE, Hoover DR, Dallabetta GA, et al. Bacterial vaginosis and disturbances of vaginal flora: associated with increased acquisition of HIV. AIDS 1998;12:1699-706.

12 Royce RA, Thorp J, Granados JL, et al. Bacterial vaginosis associated with HIV infection in pregnant women from North Carolina. F AIDS 1999;20:382-6.

13 Coggins C, Elias CJ, Atisook R, et al. A study of women's preferences regarding the formulation of over-the-counter vaginal spermicides. AIDS 1998;12:1389-91.

14 Hammet TM, Joanis CL, Mason TH, et al. Acceptability of formulations and application methods for vaginal microbicides among drug involved women: results of product trials in three cities. Abstract No 33150,12 th World AIDS
the Conference Geneva, June 28-July 31998 